\title{
Nitric oxide contributes to methane- induced osmotic stress tolerance in mung bean
}

\author{
Yihua Zhang ${ }^{1}$, Jiuchang Su${ }^{1}$, Dan Cheng ${ }^{1}$, Ren Wang ${ }^{2}$, Yudong Mei ${ }^{1}$, Huali Hu ${ }^{3}$, Wenbiao Shen ${ }^{1 *}$ \\ and Yaowen Zhang ${ }^{*}$
}

\begin{abstract}
Background: Osmotic stress is a major abiotic stress limiting crop production by affecting plant growth and development. Although previous reports discovered that methane $\left(\mathrm{CH}_{4}\right)$ has a beneficial effect on osmotic stress, the corresponding downstream signal(s) is still elusive.

Results: Polyethylene glycol (PEG) treatment progressively stimulated the production of $\mathrm{CH}_{4}$ in germinating mung bean seeds. Exogenous $\mathrm{CH}_{4}$ and sodium nitroprusside (SNP) not only triggered nitric oxide (NO) production in PEGstressed plants, but also alleviated the inhibition of seed germination. Meanwhile, amylase activity was activated, thus accelerating the formation of reducing sugar and total soluble sugar. Above responses could be impaired by $\mathrm{NO}$ scavenger(s), suggesting that $\mathrm{CH}_{4}$-induced stress tolerance was dependent on $\mathrm{NO}$. Subsequent tests showed that $\mathrm{CH}_{4}$ could reestablish redox balance in a NO-dependent fashion. The addition of inhibitors of the nitrate reductase (NR) and NO synthase in mammalian (NOS), suggested that NR and NOS-like protein might be partially involved in $\mathrm{CH}_{4}$-alleviated seed germination inhibition. In vitro and scavenger tests showed that NO-mediated S-nitrosylation might be associated with above $\mathrm{CH}_{4}$ responses.

Conclusions: Together, these results indicated an important role of endogenous $\mathrm{NO}$ in $\mathrm{CH}_{4}$-enhanced plant tolerance against osmotic stress, and NO-regulated redox homeostasis and S-nitrosylation might be involved in above $\mathrm{CH}_{4}$ action.
\end{abstract}

Keywords: Methane, Vigna radiate, Osmotic stress, Nitric oxide, Redox homeostasis

\section{Background}

Mung bean (Vigna radiata L.) is consumed in large quantities in Asia due to its desirable taste and high nutrition value [1]. It is a good source of vitamins (A, B, $\mathrm{C}$ and $\mathrm{E}$ ), minerals, and proteins with essential amino acids [2]. Mung bean has high medicinal function of curing diarrhea, headaches, edema, and eye problems [3]. However, the limited plant growth and crop production of mung bean widely exist because many regions of Asia are under osmotic stress.

\footnotetext{
* Correspondence: wbshenh@njau.edu.cn; zyw8118571@126.com ${ }^{1}$ College of Life Sciences, Laboratory Center of Life Sciences, Nanjing Agricultural University, Nanjing 210095, China

${ }^{4}$ Crop Research Institute, Shanxi Academy of Agricultural Sciences, Taiyuan 030031, China

Full list of author information is available at the end of the article
}

Osmotic stress means that the water available potential is limited. Thus, sensing and signaling during water deficit stress might play key roles in plant water status, and bring about quick changes in gene expression [4]. Generally, polyethylene glycol (PEG)-6000 is considered as an applicable solute because of its properties of mimicking osmotic stress, which results in the inhibition of plant growth and development [5]. The water deficit caused by drought or osmotic stress could usually result in many changes in plant physiological processes $[5,6]$. One of these changes is associated with the overproduction of reactive oxygen species (ROS) and thereafter oxidative damage, both of which have impacts on peroxidation of membrane lipids and the loss of plasma membrane integrity [7-9].

(c) The Author(s). 2018 Open Access This article is distributed under the terms of the Creative Commons Attribution 4.0 International License (http://creativecommons.org/licenses/by/4.0/), which permits unrestricted use, distribution, and 
More importantly, perception and transduction of the stress-induced gaseous signaling molecules, including nitric oxide (NO), hydrogen sulfide $\left(\mathrm{H}_{2} \mathrm{~S}\right)$, carbon monoxide $(\mathrm{CO})$, and hydrogen gas $\left(\mathrm{H}_{2}\right)$, are the major events [10-15]. Among these, NO is a multifunctional molecular gas, which can cross biological membranes [16]. In plants, it is considered as a regulator in response to various stresses, such as drought and osmotic stress [17, 18], salinity [11], heavy metal exposure [19], UV-B radiation [20], nanoparticles phytotoxicity [21, 22], and biotic stress $[23,24]$. Biosynthesis of NO is catalyzed by nitrate reductase (NR), a well-known route of reductive reactions, and nitric oxide synthesis (NOS)-like biochemical pathway [25, 26]. Previously, there are more evidence showed that NO could modulate ROS generation, which is always accompanied by oxidative stress, to keep redox homeostasis and decrease oxidative damage [18, 27]. Specific plant proteins involved in metabolism, stress responses, and redox homeostasis, have also been identified as possible targets for $S$-nitrosylation, one of NO-dependent post-translational modifications [28, 29].

Methane $\left(\mathrm{CH}_{4}\right)$ is not only the main element of nature gas and flammable ice, considered as a kind of clean fuel, but also have effects on anti-oxidative, anti-apoptotic and anti-inflammatory in animals [30-32]. It was further reported that formation of non-microbial $\mathrm{CH}_{4}$ in many different plant species is obviously increased by osmotic stress [33], high temperature [33, 34], UV-B radiation [33-35], physical injury [36]. Although the emission of $\mathrm{CH}_{4}$ from plant have been discovered for an extended period, the $\mathrm{CH}_{4}$ biosynthetic pathways related to $\mathrm{CH}_{4}$ formation and releasing, and its biological functions are still elusive [37]. Recent results showed that $\mathrm{CH}_{4}$ was able to induce cucumber adventitious rooting of hypocotyl cuttings (primary roots removed) [38, 39]. Similar to the response of $\mathrm{NO}$, we proved that $\mathrm{CH}_{4}$ can alleviate salinity stress and copper stress in alfalfa plants $[8,40]$. Recently, the alleviation of osmotic stress in maize seedlings by $\mathrm{CH}_{4}$ was confirmed, and the involvement of sugar and ascorbic acid metabolism was preliminarily elucidated [41]. However, the cross-talk between $\mathrm{CH}_{4}$ and $\mathrm{NO}$ signaling in plant tolerance against osmotic stress is still elusive.

To resolve above scientific question, in this study, time-course analysis of PEG-induced $\mathrm{CH}_{4}$ production was firstly determined. Considering that $\mathrm{CH}_{4}$ could form an explosive mixture with air, methane-rich water (MRW) was used to investigate the function of endogenous $\mathrm{CH}_{4}$ in plants $[8,38,39]$. Using this experimental approach, we provided pharmacological, physiological, and biochemical evidence to prove that $\mathrm{CH}_{4}$ could alleviate PEG-induced osmotic stress by modulating redox homeostasis and starch metabolism in mung bean. Importantly, this biological function was associated with the homeostasis of $\mathrm{NO}$, a key cell signaling modulator
[14-16]. The involvement of NO-triggered protein $S$-nitrosylation was also preliminarily suggested. Above results thus open a new window for $\mathrm{CH}_{4}$ signaling in plant kingdom.

\section{Methods}

\section{Chemicals}

All chemicals were purchased from Sigma (St Louis, MO, USA) unless otherwise stated. Polyethylene Glycol-6000 (PEG-6000) was purchased from Guangdong Guanghua Sci-Tech Co., Ltd, China, and is generally used to imitate osmotic stress. Sodium nitroprusside (SNP) was used as a well-known NO-releasing compound. The utterly light-inactivated SNP solution (old SNP) was used as a negative control. 2-(4-carboxyphenyl)-4,4,5,5-tetramethylimidazoline-1-oxyl-3-oxide potassium salt (cPTIO) was used as a scavenger of NO. Another scavenger of NO, 2-phenyl-4,4,5,5-tetramethylimidazoline-1-oxyl-3-oxide (PTIO) purchased from TCI company, was also used. Tungstate, a NR inhibitor, and $N^{\omega}$-nitro- ${ }_{\mathrm{L}}$-Arg methyl ester hydrochloride ( $L_{-}$NAME), a mammalian NOS inhibitor, were used, respectively. In this study, the pilot experiments were carried out to determine the suitable concentrations of above chemicals with maximal responses.

\section{Preparation of methane-rich water (MRW) and determination of methane content}

The $\mathrm{CH}_{4}$ gas $(99.9 \%$, v/v) from a compressed gas cylinder (Nanjing Special Gas Co., China) was bubbled into $500 \mathrm{ml}$ distilled water with a rate of $160 \mathrm{ml} \mathrm{min}^{-1}$ for least $30 \mathrm{~min}$ at $25^{\circ} \mathrm{C}$, thus reaching a saturated level. The corresponding methane-rich water was then immediately diluted with distilled water to different saturation required. The contents of $\mathrm{CH}_{4}$ in fresh methane-rich water $(10,50$ and $100 \%$ saturation) were $0.13,0.65$, and $1.30 \mathrm{mM}$, respectively, and maintained at original concentration for at least $12 \mathrm{~h}$.

For determining endogenous $\mathrm{CH}_{4}$ content, plant samples were treated according to the method described previously [41]. $\mathrm{CH}_{4}$ content was estimated using an Agilent 7820 model gas chromatograph (GC; Agilent Technologies Inc., USA) equipped with a flame ionization detector and a Porapak column (1/8 inch, 8 foot). The column was held isothermally at $70^{\circ} \mathrm{C}$. The injection and detector temperature was adjusted to $200{ }^{\circ} \mathrm{C}$ and $300{ }^{\circ} \mathrm{C}$, respectively. Nitrogen $\left(\mathrm{N}_{2}\right)$ was used as the carrier gas, and air pressure was 0.5 $\mathrm{MPa}$. The GC was calibrated using a standard $\mathrm{CH}_{4}$ mixture $\left(2.0 \mathrm{ppm} \mathrm{CH}_{4}\right.$ in $\mathrm{N}_{2}$; Nanjing Special Gas Co., China).

\section{Nitrogen and argon application}

For nitrogen $\left(\mathrm{N}_{2}\right)$ and argon (Ar) application, pure $\mathrm{N}_{2}$ and Ar gas from a gas cylinder (99.99\%, Nanjing special 
gas Co., Ltd) were respectively bubbled into distilled water at the same rate as $\mathrm{CH}_{4}\left(160 \mathrm{ml} \mathrm{min}^{-1}\right)$ for at least 30 min to obtain the same $\mathrm{O}_{2}$ concentration as methane-rich water (containing $1.3 \mathrm{mM} \mathrm{CH}_{4}$ ).

\section{Plant material and growth conditions}

The healthy seeds of mung bean (Vigna radiata $\mathrm{L} . \mathrm{cv}$ Jinlv No. 7) were selected and surface-sterilized with 5\% $\mathrm{NaClO}$ for $10 \mathrm{~min}$, followed by totally washed with distilled water and then dried. These seeds were presoaked with $20 \mathrm{ml}$ of culture solution containing the indicated concentrations of $\mathrm{CH}_{4}, \mathrm{~N}_{2}$, Ar, SNP, old SNP, PTIO, cPTIO, tungstate, and ${ }_{\text {L }}$ NAME, alone or their combinations. Then, these seeds were transferred to another Petri dishes and incubated on filter paper for $12 \mathrm{~h}$ at $25^{\circ} \mathrm{C}$ in the darkness following the procedure described previously $[42,43]$. The equal volume of $20 \%$ PEG- 6000 was applied to mimic osmotic stress. All seeds were germinated in a growth chamber at $25^{\circ} \mathrm{C}$ with darkness. Treatment with distilled water was regarded as control (Con). After the indicated time points, the germinating seeds were rinsed with distilled water for three times. Then, the samples were harvested and used immediately, or frozen in liquid nitrogen and stored at $-80^{\circ} \mathrm{C}$ for further analysis.

\section{Germination and growth analysis}

Germination test was carried out with three independent experiments and at least three replicates for each. Each independent set of experiments was 120 seeds. Every Petri dish contains 40 seeds. After the indicated pretreatments followed by PEG-6000 stress for $48 \mathrm{~h}$, germination rate (\%) was recorded. Seeds were regarded as germinated when the emerging root was approximately the length of the seeds. Additionally, fresh weight (FW) and dry weight (DW) were detected after various treatments for $48 \mathrm{~h}$.

\section{Detection of endogenous NO}

According to previous report [15], about $200 \mu \mathrm{m}$ transversal sections, which were obtained from root tips about $1 \mathrm{~cm}$, were cut out at the indicated time points. These sections were incubated with $7.5 \mu \mathrm{M}$ 4-amino-5-methylamino-2', $7^{\prime}$ '-difluorofluorescein diacetate (DAF-FM DA; a fairly specific NO fluorescent probe; prepared in $20 \mathrm{mM}$ HEPES buffer, $\mathrm{pH}$ 7.8) for 10 $\mathrm{min}$ at $25^{\circ} \mathrm{C}$ in the darkness. Subsequently, the sections were washed three times with the same buffer for 15 min each, and monitored by laser scanning confocal microscopy (LSCM). The DAF-FM DA signal (excitation at $488 \mathrm{~nm}$; emission at 500-530 nm) was captured as green fluorescence. All images were visualized using UltraVIEW VoX (PerkinElmer, Waltham, America). At least six individual samples were randomly selected and measured per treatment. The bright-field images corresponding to the fluorescent images were also shown. Fluorescence of NO production in root tissues (about an area of $150,000 \mu \mathrm{m}^{2}$ ) was quantified based on 20 overlapping confocal planes of $2 \mu \mathrm{m}$ each using the Volocity Demo software.

Besides, endogenous NO content was detected by Griess reagent assay [44] with some modifications. About $2000 \mathrm{mg}$ samples were homogenized in a mortar with $50 \mathrm{mM}$ cool acetic acid buffer, and then centrifuged. After various treatments, samples were pre-incubated in $100 \mu \mathrm{M} \mathrm{cPTIO}$, a specific scavenger of NO, for $1 \mathrm{~h}$, were regarded as the blank control. The supernatant was collected and reacted with Griess reagent for $30 \mathrm{~min}$. Absorbance was assayed at $540 \mathrm{~nm}$, and the NO content was calculated by in comparison with a standard curve of $\mathrm{NaNO}_{2}$.

\section{Endogenous ROS detection by LSCM}

For determining endogenous ROS, about $200 \mu \mathrm{m}$ transversal sections from the root tips were incubated with $\quad 10 \mu \mathrm{M} \quad$ 2,7'-dichlorofluorescein diacetate $\left(\mathrm{H}_{2}\right.$ DCF-DA, a fairly specific ROS fluorescent probe) [45] in $20 \mathrm{mM}$ HEPES buffer ( $\mathrm{pH} 7.8$ ) for $10 \mathrm{~min}$. The $\mathrm{H}_{2}$ DCF-DA signal (excitation at $488 \mathrm{~nm}$; emission at $500-530 \mathrm{~nm}$ ) was captured as green fluorescence, and monitored by LSCM.

\section{Histochemical staining and determination of thiobarbituric acid reactive substances (TBARS)}

After various treatments, whole roots were rinsed with distilled water for three times, and then dried with filter papers and immediately soaked in the specific reagents. Histochemical detection of lipid peroxidation was performed with Schiff's reagent [46]. All stained roots were observed under a light microscope (model Stemi 2000-C; Carl Zeiss, Germany), and photographed (Powershot A620, Canon Photo Film, Japan).

Lipid peroxidation was detected by measuring the concentration of thiobarbituric acid reactive substances (TBARS) as described previously [46]. Briefly, about $1000 \mathrm{mg}$ of germinating seeds was homogenized in a mortar with $10 \mathrm{ml}$ solution containing $0.25 \%$ 2-thiobarbituric acid (TBA) and 10\% trichloroacetic acid (TCA). After heating at $95{ }^{\circ} \mathrm{C}$ for $30 \mathrm{~min}$, the mixture was quickly cooled in an ice bath, and centrifuged at $10,000 \times \mathrm{g}$ for $10 \mathrm{~min}$. The absorbance of the supernatant was read at $532 \mathrm{~nm}$ and corrected for unspecific turbidity by subtracting the absorbance at $600 \mathrm{~nm}$. The blank was $0.25 \% \mathrm{TBA}$ in $10 \% \mathrm{TCA}$. The results were expressed as nmol $\mathrm{g}^{-1}$ fresh weight (FW). 
Determination of reducing sugar, soluble sugar content, $a$-amylase and amylase activities

For detected reducing and soluble sugar contents, $\alpha$-amylase and total amylase activities, the germinating seeds were homogenized in a mortar. Reducing sugar and soluble sugar contents were estimated following the previous methods [47]. $\alpha$-amylase and total amylase activities were detected according to the methods described previously [48].

\section{Extraction of total protein}

For protein extraction, germinating seeds were homogenized in a mortar with liquid nitrogen to fine powder, and then re-suspended in HEN buffer containing 250 $\mathrm{mM}$ Hepes- $\mathrm{NaOH}$ (pH 7.7), $1 \mathrm{mM}$ EDTA, and $0.1 \mathrm{mM}$ protease inhibitor cocktail, and centrifuged at 13,000 g for $20 \mathrm{~min}$ at $4^{\circ} \mathrm{C}$. The supernatants were transferred to clean tube stored at $4^{\circ} \mathrm{C}$ for Western blotting analysis.

\section{Western blotting analyses of protein S-nitrosylation}

According to the previous protocols [39], analysis of protein $S$-nitrosylation was carried out. The biotin-labeled protein samples were separated under non-reducing conditions by $12 \%$ SDS-PAGE for $1.5 \mathrm{~h}$ at $120 \mathrm{~V}$. After blotting onto a polyvinylidene difluoride (PVDF) membrane, anti-biotin antibody (HRP; Abcam antibodies, Cambridge, UK) was added at 1:10,000 dilution for $1 \mathrm{~h}$ at room temperature. Meanwhile, as a loading control, parallel sets of gels were stained with colloidal Coomassie blue to confirm that the loaded proteins were equal amounts.

\section{Statistical analyses}

All date expressed are the mean values \pm SE of three independent experiments with at least three replicates for each. Statistical analysis was performed using SPSS 16.0 software. For statistical analysis, one-way analysis of variance (ANOVA) followed by Duncan's multiple range test $(P<0.05)$ was chosen.

\section{Results}

\section{Osmotic stress induces $\mathrm{CH}_{4}$ production in a} time-dependent fashion

In order to evaluate whether osmotic stress could induce the production of $\mathrm{CH}_{4}, \mathrm{CH}_{4}$ content was analyzed in germinating mung bean seeds by gas chromatography (GC). Fig. 1 showed that, in comparison with control samples, PEG stress resulted in a gradual and significant increase in $\mathrm{CH}_{4}$ production during a $48 \mathrm{~h}$ period of treatment, suggesting the possible role of endogenous $\mathrm{CH}_{4}$ in osmotic stress responses.

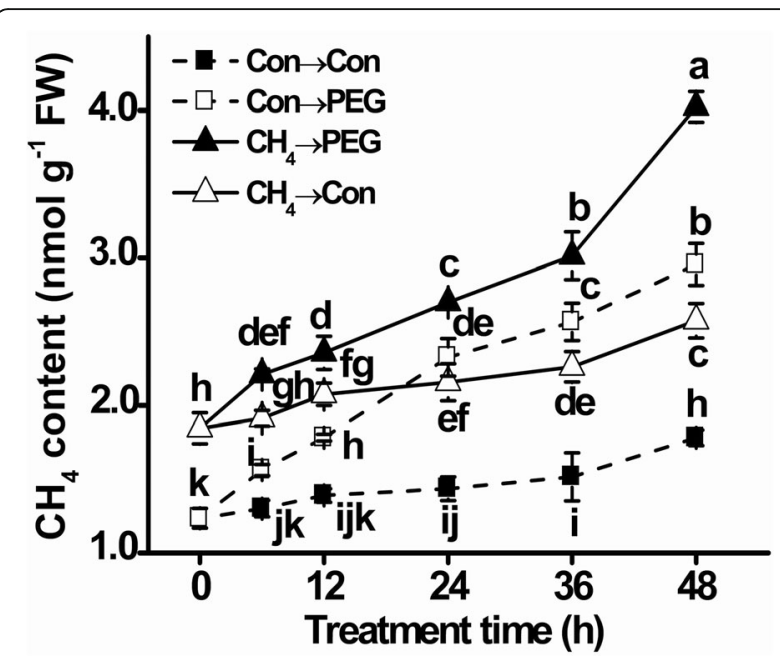

Fig. 1 Time-dependent changes of $\mathrm{CH}_{4}$ production. Mung bean seeds presoaked with solution containing $1.3 \mathrm{mM} \mathrm{CH}_{4}$ for $12 \mathrm{~h}$, were then shifted to $20 \%$ PEG-6000 for another $48 \mathrm{~h}$. The endogenous $\mathrm{CH}_{4}$ production in germinating seeds was then detected by gas chromatography (GC). Treatment with distilled water was regarded as control (Con). Data are means \pm SE of three independent experiments with three replicates for each. Bars with different letters denote significant difference at $P<0.05$ according to Duncan's multiple range test

PEG-induced inhibition of seed germination was alleviated by $\mathrm{CH}_{4}$ and sodium nitroprusside (SNP) To characterize the effect of $\mathrm{CH}_{4}$ on osmotic stress, culture medium containing different concentrations of $\mathrm{CH}_{4}$ (ranging from 0.13 to $1.30 \mathrm{mM}$ ) were applied. As showed in Fig. 2a, the inhibition of seed germination was observed in PEG-treated sample. Further results illustrated that $\mathrm{CH}_{4}$ pretreatment was effective in reversing the negative impact of PEG stress on seed germination in a dose-dependent manner, with $1.30 \mathrm{mM}$ in particular.

Results shown in Fig. 1 further revealed that $1.3 \mathrm{mM}$ $\mathrm{CH}_{4}$ pretreatment for $12 \mathrm{~h}(0 \mathrm{~h}$; the beginning of osmotic stress) brought about a $49.2 \%$ increase in $\mathrm{CH}_{4}$ production. Subsequent stress $\left(\mathrm{CH}_{4} \rightarrow \mathrm{PEG}\right)$ aggravated $\mathrm{CH}_{4}$ production, compared to stress alone. Based on the above findings, $1.3 \mathrm{mM} \mathrm{CH}_{4}$ was used in the subsequent experiment.

To rule out the possibility that $\mathrm{CH}_{4}$-promoted role might be partly due to hypoxia, culture medium containing nitrogen gas $\left(\mathrm{N}_{2}\right)$ and inert gas argon (Ar) was subsequently applied. As expected, unlike the beneficial response of $\mathrm{CH}_{4}$, both $\mathrm{N}_{2}$ and Ar failed to alleviate PEG-triggered seed germination inhibition (Fig. 2b).

It was also noticed that the application of sodium nitroprusside (SNP; a NO-releasing compound) (Fig. 3), brought about the significant alleviation in the seed germination inhibition caused by PEG stress. Above response was not observed in the pretreatment with old 

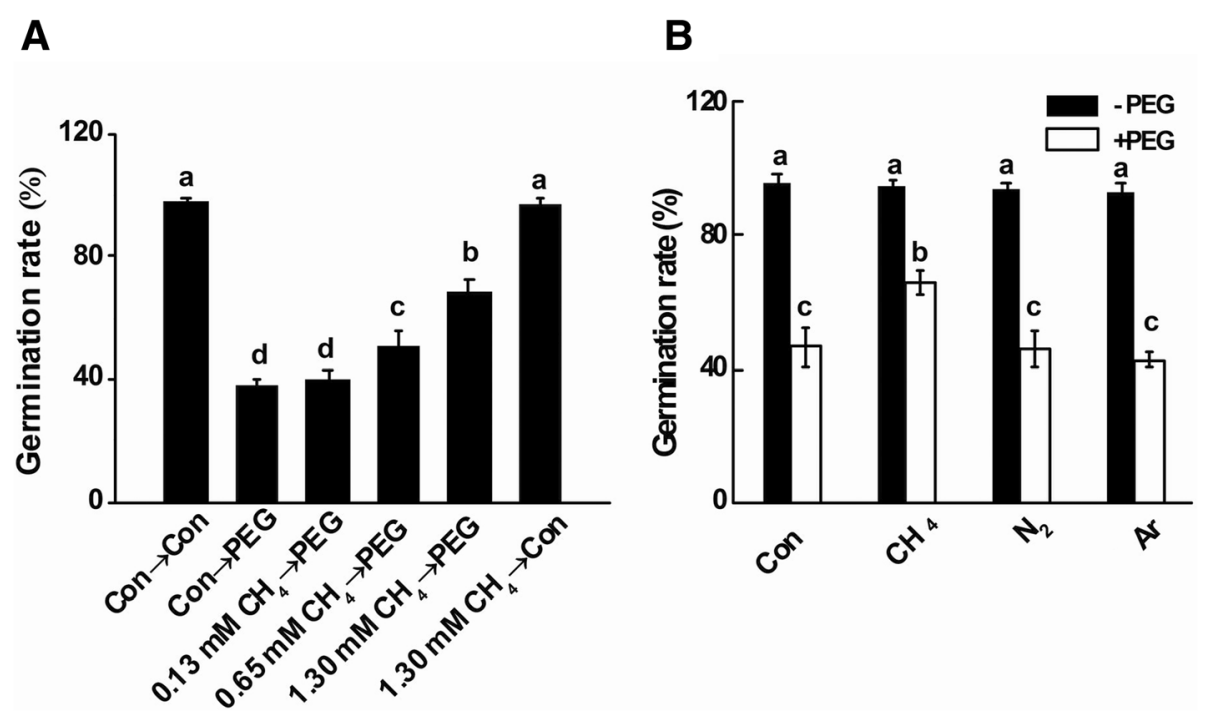

Fig. 2 Unlike the responses of $\mathrm{CH}_{4}$ (a), hypoxia (b) failed to alleviate inhibition of seed germination caused by PEG. Mung bean seeds presoaked with solutions containing the indicated concentrations of $\mathrm{CH}_{4}$, nitrogen $\left(\mathrm{N}_{2}\right)$, or argon (Ar) for $12 \mathrm{~h}$, were then shifted to 20\% PEG-6000 for another $48 \mathrm{~h}$. Afterwards, germination rates were determined. Treatment with distilled water was regarded as control (Con). Data are means \pm SE of three independent experiments with at least three replicates for each. Bars with different letters denote significant difference at $P<0.05$ according to Duncan's multiple range test

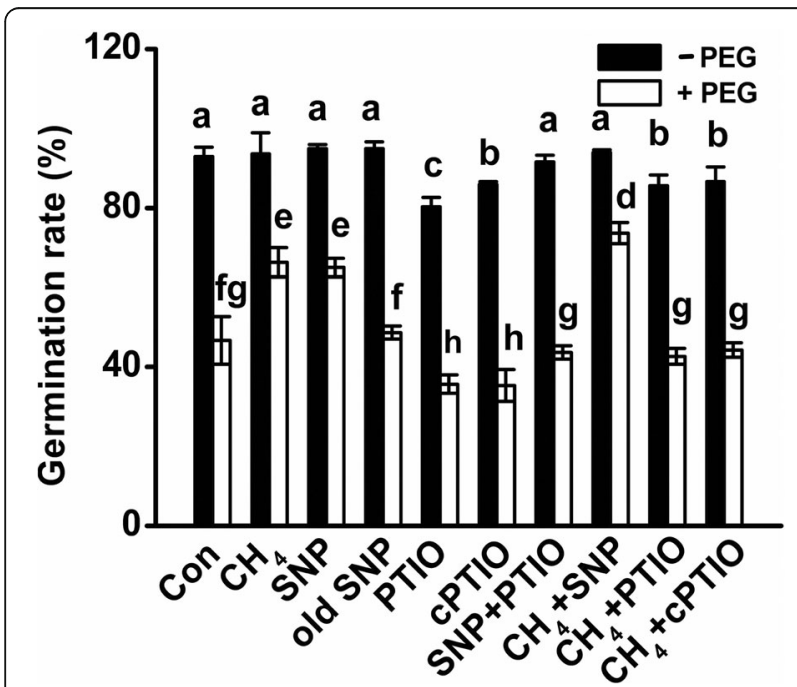

Fig. $3 \mathrm{CH}_{4}$-induced alleviation of seed germination inhibition caused by PEG stress was sensitive to PTIO and CPTIO. Mung bean seeds presoaked with solutions containing $1.3 \mathrm{mM} \mathrm{CH}_{4}, 100 \mu \mathrm{M}$ SNP, $100 \mu \mathrm{M}$ old SNP, $500 \mu \mathrm{M}$ PTIO, $500 \mu \mathrm{M}$ CPTIO, alone or their combinations for $12 \mathrm{~h}$, were then shifted to 20\% PEG-6000 for another $48 \mathrm{~h}$. Afterwards, germination rates were determined. Treatment with distilled water was regarded as control (Con). Data are means \pm SE of three independent experiments with at least three replicates for each. Bars with different letters denote significant difference at $P<0.05$ according to Duncan's multiple range test
SNP solution (a negative control of SNP, containing no $\mathrm{NO}$, but ferrocyanide, nitrate and nitrite), suggesting that the beneficial role of SNP was NO-dependent.

\section{$\mathrm{CH}_{4}$-alleviated seed germination inhibition was sensitive to CPTIO and PTIO, two scavengers of NO}

To investigate the possible involvement of $\mathrm{NO}$ in above $\mathrm{CH}_{4}$ response, two specific scavengers of $\mathrm{NO}$, 2-phenyl-4,4,5,5-tetramethylimidazoline-1-oxyl-3-oxide (PTIO) and 2-(4-carboxyphenyl)-4,4,5,5-tetramethylimidazoline-1-oxyl-3-oxide potassium salt (cPTIO), were used. Interestingly, we observed that $\mathrm{CH}_{4}$-induced alleviation of seed germination inhibition was significantly blocked by the addition of cPTIO and PTIO, respectively (Fig. 3). The cotreatment with PTIO suppressed the inducible effect of SNP on the alleviation of seed germination inhibition as well. The additive behavior appeared when $\mathrm{CH}_{4}$ was added together with SNP followed by stress. These results, together with the performances of old SNP (Fig. 3), suggested the importance role of NO in the beneficial responses triggered by $\mathrm{CH}_{4}$. In view of the relative expensive price of CPTIO and higher amount of scavenger required for a fully assay, we adopted PTIO as a NO scavenger in the subsequent investigation.

\section{NO might be involved in $\mathrm{CH}_{4}$-modulated starch metabolism in response to osmotic stress}

To investigate the mechanism of $\mathrm{CH}_{4}$-alleviated seed germination inhibition, several physiological parameters, including reducing and soluble sugar contents, $\alpha$-amylase 
and total amylase activities, were detected. As shown in Table 1, PEG stress for $48 \mathrm{~h}$ resulted in obvious decline of those parameters in germinating seeds. The combination of PEG with $\mathrm{CH}_{4}$ or SNP pretreatment enhanced $\alpha$-amylase and total amylase activities, both of which were in accordance with the increased contents of reducing sugar and soluble sugar. On the contrary, above changes in starch metabolism triggered by $\mathrm{CH}_{4}$ and SNP were impaired by the addition of PTIO. Alone, PTIO decreased above parameters, suggesting the possible role of endogenous NO.

\section{$\mathrm{CH}_{4}$-mediated endogenous $\mathrm{NO}$ generation was reversed} by tungstate and L-NAME, two synthetic inhibitors of NO In order to assess the role of $\mathrm{NO}$ in the physiological role of $\mathrm{CH}_{4}$, endogenous $\mathrm{NO}$ levels in root tissues were checked by using the permeable NO-sensitive fluorophore 4-amino-5-methylamino-2'7'-difluorofluorescein diacetate (DAF-FM DA) in combination with laser scanning confocal microscopy (LSCM). We compared fluorescence detected in the presence of SNP, old SNP, and PTIO. As expected, in the presence (in particularly) or absence of PEG stress conditions, SNP-induced fluorescence was differentially reduced when root tissues were co-pretreated with PTIO (Fig. 4a, b). Unlike SNP, old SNP failed to influence PEG-induced DAF-FM DA green fluorescence.

Under osmotic stress conditions, the $\mathrm{CH}_{4}$-triggered induction of DAF-FM DA fluorescence was markedly abolished by the removal of NO with PTIO, suggesting that above strengthened-fluorescence was $\mathrm{NO}$-specific (Fig. 4a, b). The obtained data with Griess reagent assay (Fig. 4c) were in line with those of DAF-FM-associated fluorescence, further confirming that the DAF-FMdependent fluorescence was related to NO levels in vivo. Combined with corresponding phenotypes (Fig. 3), these result apparently supported the idea that $\mathrm{NO}$ production might be involved in $\mathrm{CH}_{4}$-induced tolerance against PEG stress.

To better characterize the main source(s) of NO generated by $\mathrm{CH}_{4}$ in stressed mung bean, seeds were pretreated with chemicals that interfere with $\mathrm{NO}$ production before osmotic stress. In our experiment, tung-

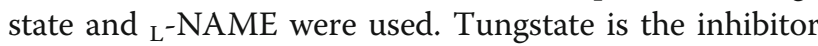
of NR [15], and ${ }_{L}$-NAME is the inhibitor of mammalian NOS, which was usually applied to inhibit plant NOS-like activity [39, 49]. As shown in Fig. 4d, tungstate significantly blocked $\mathrm{CH}_{4}$-alleviated seed germination inhibition, suggesting that $\mathrm{CH}_{4}$-triggered $\mathrm{NO}$ production might be partly resulted from NR. Comparatively, L-NAME had a lesser, yet significant influence on the $\mathrm{CH}_{4}$-triggerd response, implying that NOS-like enzyme might be partly involved.

\section{$\mathrm{CH}_{4}$-modualted redox homeostasis was sensitive to the removal of endogenous NO}

Upon stress conditions, redox homeostasis is impaired, and NO-mediated plant tolerance against stress is normally associated with the reestablishment of redox homeostasis [50]. To further investigate the mechanism of $\mathrm{CH}_{4}$-elicited tolerance against osmotic stress, we analyzed whether redox homeostasis was involved, in a manner similar to NO response. Sections detached from root tips were stained with $\mathrm{H}_{2}$ DCF-DA (a probe for ROS), and LSCM was used to check changes in intracellular ROS levels (Fig. 5a, b). As expected, ROS overproduction

Table 1 Both $\mathrm{CH}_{4}$ - and NO-alleviated sugar metabolism inhibition caused by PEG stress were sensitive to PTIO

\begin{tabular}{|c|c|c|c|c|}
\hline Treatments & $\begin{array}{l}\text { Reducing sugar content } \\
\left(\mathrm{mg} \mathrm{g}^{-1} \mathrm{DW}\right)\end{array}$ & $\begin{array}{l}\text { Soluble sugar content } \\
\left.\text { (mg g }{ }^{-1} \mathrm{DW}\right)\end{array}$ & $\begin{array}{l}a \text {-amylase activity } \\
\left(\mathrm{mg} \mathrm{min}^{-1} \mathrm{~g}^{-1} \mathrm{DW}\right)\end{array}$ & $\begin{array}{l}\text { Amylase activity } \\
\left(\text { mg } \text { min }^{-1} \mathrm{~g}^{-1} \mathrm{DW}\right)\end{array}$ \\
\hline Con $\rightarrow$ Con & $31.76 \pm 1.80^{b}$ & $18.60 \pm 0.37^{b}$ & $68.36 \pm 5.16^{b}$ & $429.37 \pm 14.11^{\mathrm{b}}$ \\
\hline Con $\rightarrow$ PEG & $20.71 \pm 0.77^{f}$ & $14.25 \pm 0.77^{f}$ & $21.28 \pm 2.18^{f}$ & $173.68 \pm 4.19^{f}$ \\
\hline $\mathrm{CH}_{4} \rightarrow \mathrm{PEG}$ & $28.22 \pm 0.45^{d}$ & $16.77 \pm 0.32^{d}$ & $35.16 \pm 3.25^{d}$ & $275.48 \pm 7.84^{d}$ \\
\hline $\mathrm{CH}_{4} \rightarrow \mathrm{Con}$ & $34.02 \pm 0.78^{\mathrm{a}}$ & $21.11 \pm 0.56^{\mathrm{a}}$ & $79.40 \pm 5.05^{a}$ & $474.73 \pm 16.27^{a}$ \\
\hline $\mathrm{CH}_{4}+\mathrm{PTIO} \rightarrow \mathrm{PEG}$ & $26.65 \pm 0.47^{e}$ & $15.85 \pm 0.33^{e}$ & $25.06 \pm 2.83^{e}$ & $219.84 \pm 2.07^{e}$ \\
\hline $\mathrm{CH}_{4}+\mathrm{PTIO} \rightarrow \mathrm{Con}$ & $30.87 \pm 0.51^{b}$ & $18.29 \pm 0.28^{b}$ & $63.14 \pm 4.99^{b c}$ & $412.25 \pm 47.67^{\mathrm{bc}}$ \\
\hline $\mathrm{SNP} \rightarrow \mathrm{PEG}$ & $28.67 \pm 0.86^{d}$ & $16.50 \pm 0.82^{d}$ & $38.82 \pm 1.85^{d}$ & $280.53 \pm 10.74^{d}$ \\
\hline $\mathrm{SNP} \rightarrow \mathrm{Con}$ & $34.32 \pm 1.88^{\mathrm{a}}$ & $21.32 \pm 1.34^{\mathrm{a}}$ & $84.54 \pm 3.93^{\mathrm{a}}$ & $488.89 \pm 11.33^{\mathrm{a}}$ \\
\hline $\mathrm{SNP}+\mathrm{PTIO} \rightarrow \mathrm{PEG}$ & $26.18 \pm 1.19^{e}$ & $15.52 \pm 0.05^{e}$ & $26.30 \pm 4.20^{\mathrm{e}}$ & $233.44 \pm 7.07^{e}$ \\
\hline $\mathrm{SNP}+\mathrm{PTIO} \rightarrow$ Con & $30.37 \pm 1.01^{b}$ & $18.37 \pm 0.93^{b}$ & $62.00 \pm 4.81^{b c}$ & $412.83 \pm 46.29^{b c}$ \\
\hline $\mathrm{PTIO} \rightarrow \mathrm{PEG}$ & $18.86 \pm 0.99^{9}$ & $12.74 \pm 0.55^{\mathrm{g}}$ & $12.87 \pm 3.01^{\mathrm{g}}$ & $127.62 \pm 9.15^{9}$ \\
\hline $\mathrm{PTIO} \rightarrow$ Con & $29.94 \pm 0.59^{c}$ & $17.28 \pm 0.52^{c}$ & $60.43 \pm 0.50^{c}$ & $393.47 \pm 10.31^{c}$ \\
\hline
\end{tabular}

Mung bean seeds were presoaked with solutions containing $1.3 \mathrm{mM} \mathrm{CH}_{4}, 100 \mu \mathrm{M} \mathrm{SNP}, 500 \mu \mathrm{M} \mathrm{PTIO}$, alone or their combinations for $12 \mathrm{~h}$, and then shifted to $20 \%$ PEG-6000 for another $48 \mathrm{~h}$. Afterwards, reducing and soluble sugar contents, $a$-amylase and total amylase activities in germinating seeds, were determined. Treatment with distilled water was regarded as control (Con). Within each set of experiments, data are means \pm SE of three independent experiments with at least three replicates for each. Bars with different letters denote significant difference at $P<0.05$ according to Duncan's multiple test 


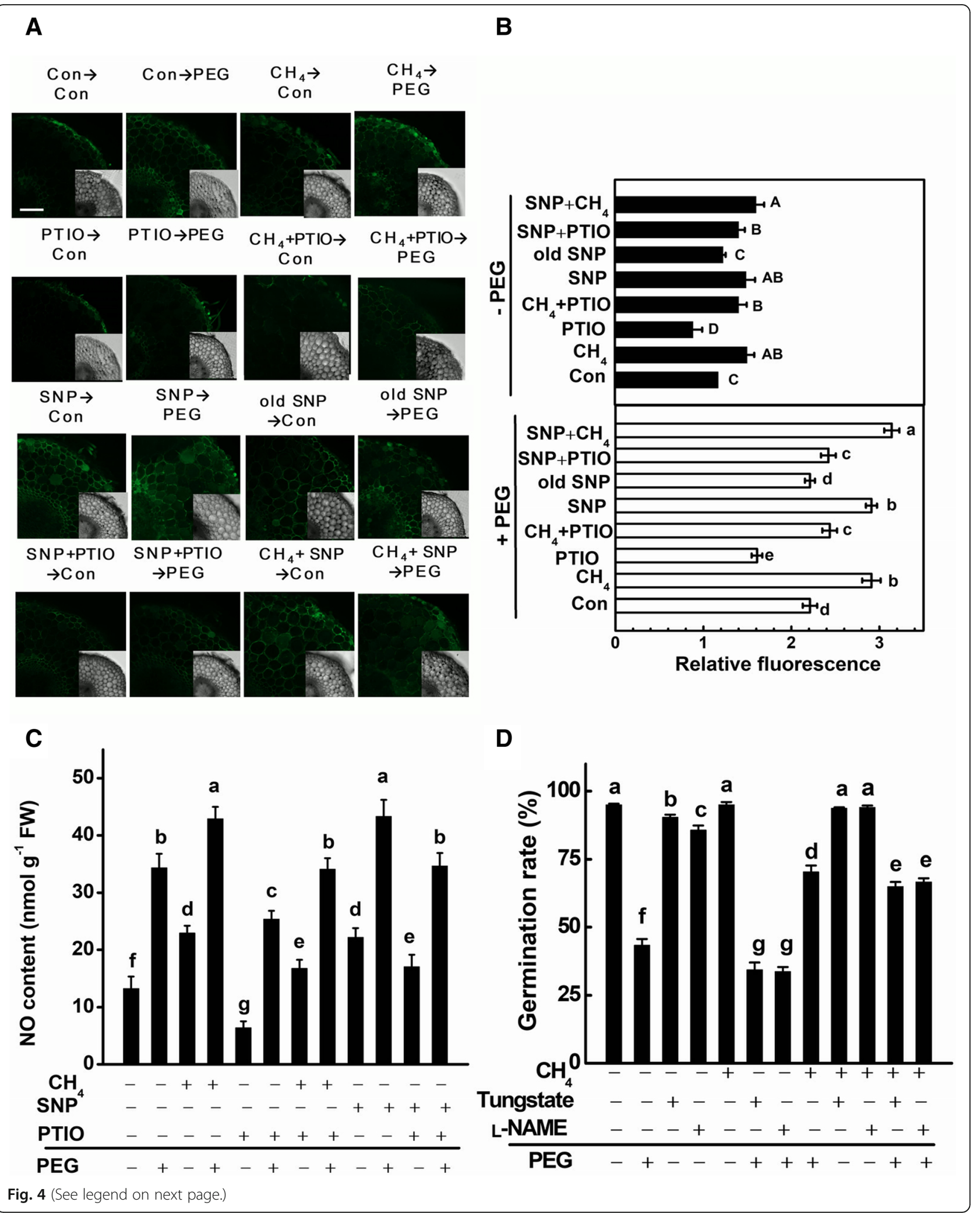




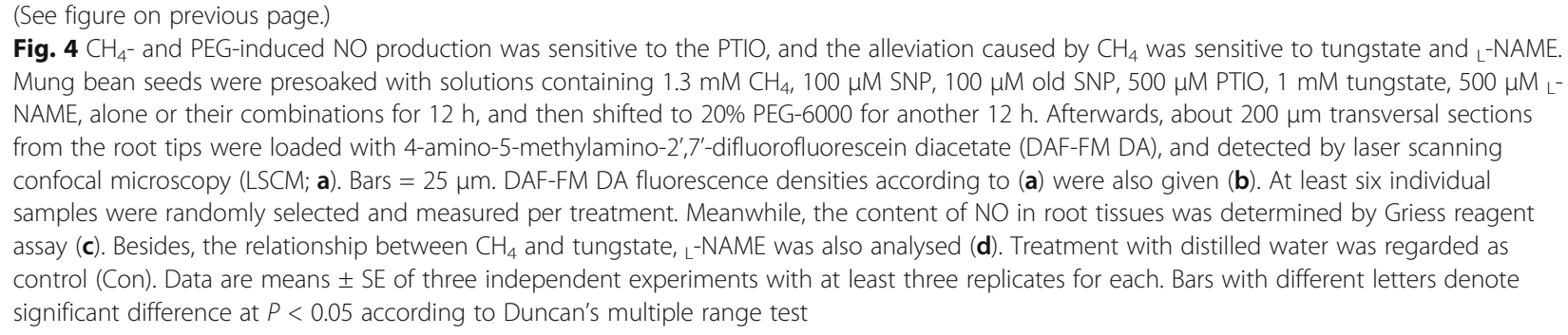

occurred when PEG was supplemented, confirming that redox imbalance happened. PTIO alone induced redox imbalance as well. Further results revealed that PEG-triggered redox imbalance was markedly blocked by the addition of $\mathrm{CH}_{4}$, which was abolished by PTIO. Similar responses were observed when SNP was applied. Above results suggested that $\mathrm{CH}_{4}$-modualted redox homeostasis was sensitive to the removal of endogenous NO.

To confirm above deduction, a histochemical staining by Schiff's reagent, which is used to monitor the level of peroxidation of membrane lipids, was performed (Fig. 5c). Compared with the control samples, the roots of mung bean treated with PEG alone or pretreated with PTIO alone were stained extensively. Those pretreated with $\mathrm{CH}_{4}$ or SNP followed by stress showed a less staining, which were markedly reversed when PTIO was cotreated together. Meanwhile, changes in TBARS contents exhibited the similar tendencies (Fig. 5d).

\section{The possible involvement of $\mathrm{CH}_{4}$-triggered NO-mediated S-nitrosylation}

To further understand the possible role of $\mathrm{CH}_{4}$ in protein level, the NO-mediated $S$-nitrosylated protein level was detected by using the protein extracted from mung bean and the modified biotin switch assay. Fig. 6 showed that similar to the responses of SNP alone, stress stimulated nitrosylation, which was strengthened by $\mathrm{CH}_{4}$ or SNP. By contrast, above $\mathrm{CH}_{4}$ - or SNP-stimulated nitrosylation in stressed plants were obviously abolished when endogenous NO was removal with PTIO. Alone, the pretreatment with PTIO could decrease nitrosylation levels in the presence or absence of PEG.

\section{Discussion}

Here, we provided the molecular basis of $\mathrm{CH}_{4}$-induced plant tolerance against osmotic stress: the involvement of NO signaling.

Similar to our previous findings in maize root tissues upon osmotic stress [41], this report revealed that an increase in the concentration of $\mathrm{CH}_{4}$ is one of the earliest responses involved in the signaling cascade triggered by PEG stress in germinating mung bean seeds (Fig. 1). Although the biochemical routes responsible for plant
$\mathrm{CH}_{4}$ production have not elucidated in this report, our finding that PEG triggered $\mathrm{CH}_{4}$ production was in agreement with those obtained in germinating alfalfa seeds subjected to copper stress [40] and salinity [8]. Combined with the results showing that osmotic stress obviously increased $\mathrm{CH}_{4}$ emission in pea leaves [33], and $\mathrm{CH}_{4}$ might be associated with adventitious rooting in $\mathrm{cu}$ cumber [38,39], we therefore deduced that $\mathrm{CH}_{4}$ might be produced and then emitted by plants as a consequence of osmotic stress, and this might be a universal event, or stress- and even developmental stage-specific in different plant species. The possible role(s) of endogenous $\mathrm{CH}_{4}$ production was subsequently investigated in PEG-stressed mung bean.

The physiological function of $\mathrm{CH}_{4}$ was firstly recognized in animals [31], showing that it displays the protective response against the intestinal ischemic/reperfusion (IR) injury-induced oxidative stress and inflammation. Until now, it was recognized that $\mathrm{CH}_{4}$ serves as multiple functions in animals via anti-oxidative, anti-apoptotic and anti-inflammatory actions $[30,32,51]$. Similar to the beneficial roles of $\mathrm{NO}$ against osmotic stress and drought in plants $[17,18]$, by using culture solutions containing different concentrations of $\mathrm{CH}_{4}$, we provided evidence, showing that $\mathrm{CH}_{4}$ could participate in the promotion of plant tolerance against osmotic stress in mung bean during germination.

First, PEG-induced inhibition of seed germination was alleviated by $\mathrm{CH}_{4}$ in a dose-dependent fashion, with 1.3 $\mathrm{mM}$ in maximum response (Fig. 2a). Above beneficial role could be explained by $\mathrm{CH}_{4}$-induced $\alpha$-amylase and total amylase activities, thus accelerating the formation of reducing and total sugar (Table 1). These results were consistent with our recent report [41], showing that PEG-induced $\mathrm{CH}_{4}$ production was more pronounced in the drought stress-tolerant maize cultivar than stress-sensitive cultivar. Our subsequent experiments confirmed that the main factor of $\mathrm{CH}_{4}$-rich solution responsible for the alleviation of seed germination inhibition was the dissolved $\mathrm{CH}_{4}$, rather than hypoxia (Fig. 2b). Similar results were confirmed in hydrogen-rich water-mediated tolerance against drought in Arabidopsis [45]. In view of the fact that the inhibition of seed germination is one of the representative phenotypes in response to osmotic 


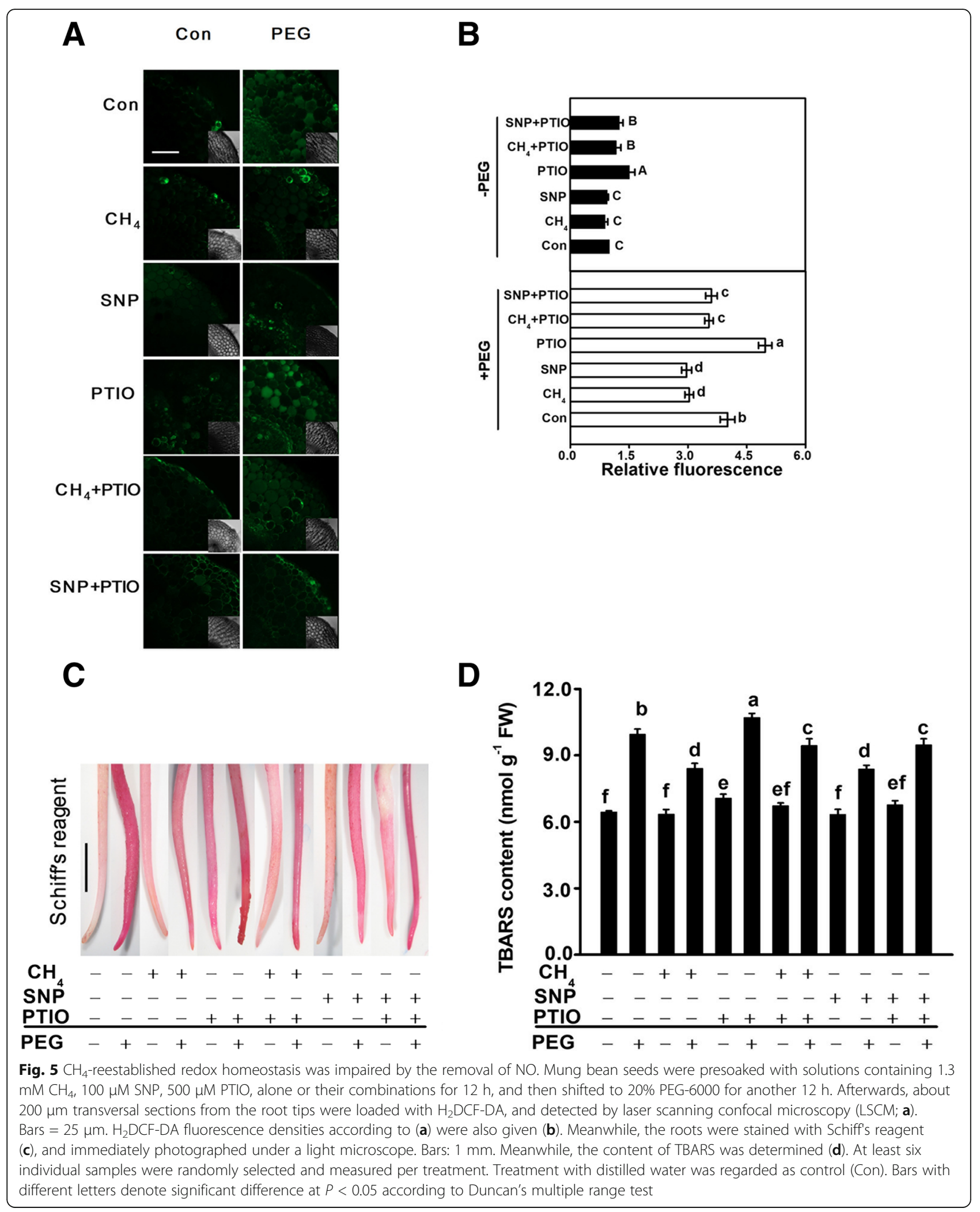



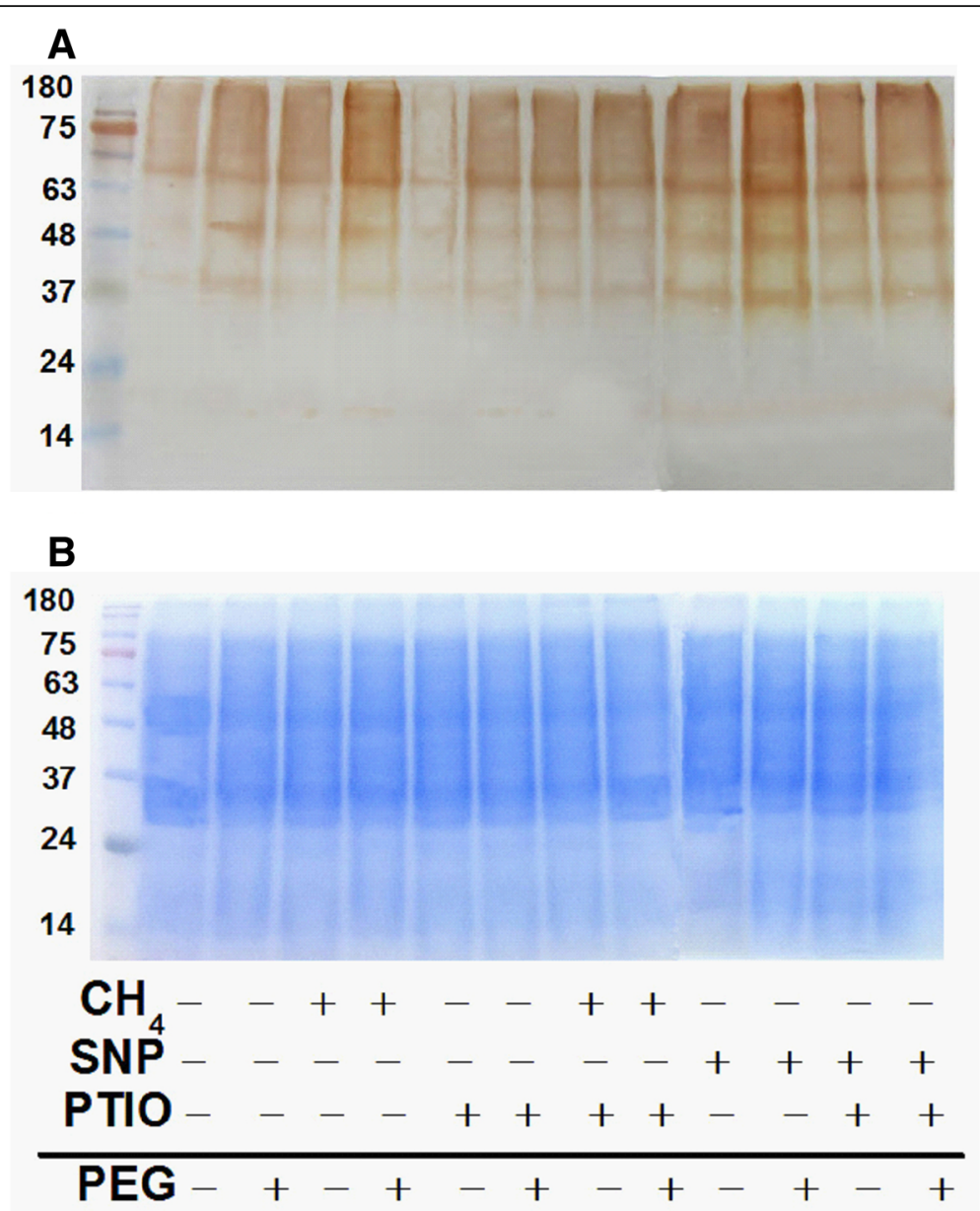

Fig. 6 In vivo analysis revealed that $\mathrm{CH}_{4}$-mediated S-nitrosylation was sensitive to PTIO. Mung bean seeds were presoaked with solutions containing $1.3 \mathrm{mM} \mathrm{CH}_{4}, 100 \mu \mathrm{M} \mathrm{SNP}, 500 \mu \mathrm{M} \mathrm{PTIO}$, alone or their combinations for $12 \mathrm{~h}$, and then shifted to 20\% PEG-6000 for another $48 \mathrm{~h}$. Biotin labeled proteins were detected by Western blot with anti-biotin antibodies (a). Meanwhile, the equal protein amounts were evaluated by Coomassie staining (b) after SDS-PAGE separation. Numbers on the left of the panels indicate the position of the protein markers in $\mathrm{kDa}$. The gels are representative of at lest five replicates per experiment

stress, we therefore confirmed that $\mathrm{CH}_{4}$ enhances plant tolerance against osmotic stress. Similar rescuing responses in salinity [8] and heavy metal stress [40] were previously reported, although the specific mechanisms are still not fully elucidated.

It has been well documented that gaseous signaling molecules have not only discrete, but also overlapping roles in conferring plant stress tolerance [11, 17, 25]. In the subsequent work, we confirmed that the beneficial roles of $\mathrm{CH}_{4}$ are, at least partly, dependent on the action of NO, a well-known gaseous signaling molecule in plants $[16,23]$. First, $\mathrm{CH}_{4}$ strengthened the increase in $\mathrm{NO}$ production in roots upon PEG stress (Fig. $4 \mathrm{a}-\mathrm{C}$ ). Above mentioned $\mathrm{CH}_{4}$-triggered $\mathrm{NO}$ production was markedly impaired by the addition of PTIO (a scavenger of NO), which was confirmed by the combination of LSCM and Griess reagent assay. Similar phenomenon occurred when SNP was applied in the presence of PEG and PTIO. Meanwhile, related phenotypes in terms of the alleviation of seed germination inhibition (Fig. 3) and corresponding parameters (Table 1) were reversed. Third, unlike the responses of SNP, old SNP failed to influence above parameters. These results, together with our previous results [39], suggested the novel function of NO in the beneficial roles of $\mathrm{CH}_{4}$ in stressed conditions and different developmental processes. Additionally, the possible involvement of NR and NOS-like protein in $\mathrm{CH}_{4}$-induced NO production was preliminarily corroborated by the findings that corresponding inhibitors (tungstate and L-NAME) inhibited $\mathrm{CH}_{4}$-alleviated seed germination inhibition caused by PEG (Fig. 4d). In fact, the contribution of NR is very difficult to evaluate in our experimental conditions, since tungstate, an inhibitor of NR, is also a ABA synthetic inhibitor. Thus, further genetic evidence should be considered. Certainly, other route(s) responsible for $\mathrm{NO}$ production might be another consideration. 


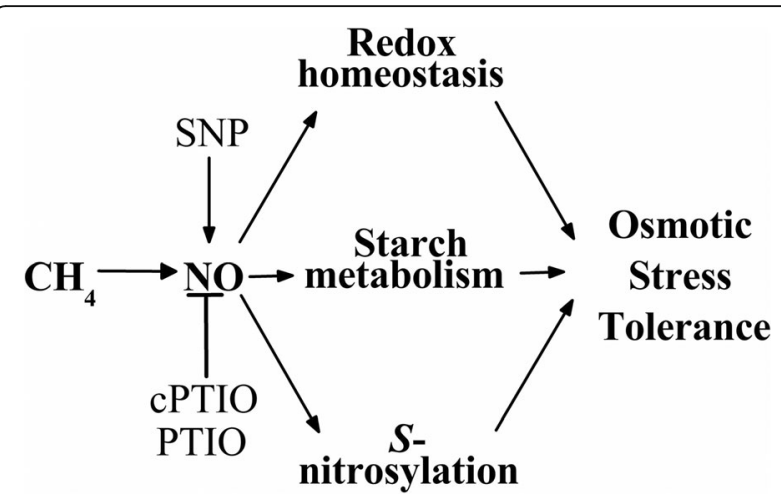

Fig. 7 Schematic representation of the signaling pathway involving $\mathrm{CH}_{4}, \mathrm{NO}$, reestablishment of redox homeostasis, starch metabolism and S-nitrosylation, during osmotic stress tolerance. The signaling cascade showed that the beneficial role of $\mathrm{CH}_{4}$ was in a NOdependent fashion. T bar, inhibition

Keeping redox homeostasis is an important mechanism for plant tolerance against osmotic stress [7]. Upon PEG stress, redox imbalance occurred. For instances, PEG stress could obviously induce ROS overproduction (Fig. 5a, b) and oxidative damage (Fig. 5c, d). These responses could be alleviated by $\mathrm{CH}_{4}$. It was further observed that $\mathrm{CH}_{4}$-reestablished redox balance was NO-dependent, since PTIO could counteract the effect of $\mathrm{CH}_{4}$. Together, above results indicated that $\mathrm{CH}_{4}$-evoked endogenous $\mathrm{NO}$ production in mung bean was positively correlated with plant tolerance against osmotic stress, and the reestablishment of redox homeostasis was an important mechanism.

It was well-known that NO-based $S$-nitrosylation is a highly conserved protein posttranslational modification that regulates diverse biological processes [39]. To further confirm the role of $\mathrm{NO}$ in the actions of $\mathrm{CH}_{4}$, the combination with pharmacological approach and biotin switch method was used (Fig. 6). Consistent with the previous results in cucumber explants [39], our results suggested the role of NO-mediated $S$-nitrosylation in $\mathrm{CH}_{4}$ responses, which might be in a stress- and development-specific fashion [28].

\section{Conclusion}

Taken together, our results define a main branch of NO-regulated redox homeostasis and starch metabolism involved in the $\mathrm{CH}_{4}$ signaling cascade during plant tolerance against osmotic stress (Fig. 7). $\mathrm{CH}_{4}$-governed NO-mediated $S$-nitrosylation might be an interesting mechanism. Therefore, the identification of NO-targeted nitrosylated protein(s) by using nanoLC/MS/MS might help us to understand the detailed mechanism of $\mathrm{CH}_{4}$ action.

\section{Abbreviations}

Ar: Argon; $\mathrm{CH}_{4}$ : methane; $\mathrm{CO}$ : Carbon monoxide; CPTIO: 2-(4-carboxyphenyl)4,4,5,5-tetramethylimidazoline-1-oxyl-3-oxide potassium salt; DAF-FM DA: 4amino-5-methylamino-2',7'-difluorofluorescein diacetate; DW: Dry weight; FW: Fresh weight; GC: Gas chromatograph; $\mathrm{H}_{2}$ : hydrogen gas; $\mathrm{H}_{2} \mathrm{DCF}-$ DA: 2',7'-dichlorofluorescein diacetate; $\mathrm{H}_{2} \mathrm{~S}$ : hydrogen sulfide; L-NAME: $N^{\omega}$ nitro-_-Arg methyl ester hydrochloride; LSCM: Laser scanning confocal microscopy; MRW: Methane-rich water; $\mathrm{N}_{2}$ : Nitrogen; NO: Nitric oxide; NOS: Nitric oxide synthesis; NR: Nitrate reductase; PEG: Polyethylene glycol; PTIO: 2-phenyl-4,4,5,5-tetramethylimidazoline-1-oxyl-3-oxide;

PVDF: Polyvinylidene difluoride; ROS: Reactive oxygen species; SNP: Sodium nitroprusside; TBA: 2-thiobarbituric acid; TBARS: Thiobarbituric acid reactive substances; TCA: Trichloroacetic acid

\section{Funding}

This work was financially supported by the Postgraduate Research \& Practice Innovation Program of Jiangsu Province (KYCX17_0660), the Fundamental Research Funds for the Central Universities (KYTZ201402), the China Agriculture Research System (CARS-08), and the Priority Academic Program Development of Jiangsu Higher Education Institutions (PAPD).

\section{Availability of data materials}

All data generated or analyzed during this study are included in this published article.

\section{Authors' contributions}

$Y Z^{1}$, WS and $Y Z^{2}$ conceived and designed the study. $Y Z^{1}, J S, D C, R W, Y M$ and $\mathrm{HH}$ participated in acquisition of data for the study. $Y Z^{1}, W S$ and $Y Z^{2}$

analyzed the data. All authors read and approved the final manuscript.

Ethics approval and consent to participate

Not applicable.

\section{Consent for publication}

Not applicable.

\section{Competing interests}

The authors declare that they have no competing interests.

\section{Publisher's Note}

Springer Nature remains neutral with regard to jurisdictional claims in published maps and institutional affiliations.

\section{Author details}

${ }^{1}$ College of Life Sciences, Laboratory Center of Life Sciences, Nanjing Agricultural University, Nanjing 210095, China. ${ }^{2}$ Institute of Botany, Jiangsu Province and Chinese Academy of Sciences, Nanjing 210014, China. ${ }^{3}$ Institute of Agricultural Products Processing, Jiangsu Academy of Agricultural Sciences, Nanjing 210014, China. ${ }^{4}$ Crop Research Institute, Shanxi Academy of Agricultural Sciences, Taiyuan 030031, China.

Received: 11 April 2018 Accepted: 16 September 2018 Published online: 24 September 2018

\section{References}

1. Peñas E, Gómez R, Frías J, Vidal-Valverde C. Effects of combined treatments of high pressure, temperature and antimicrobial products on germination of mung bean seeds and microbial quality of sprouts. Food Control. 2010;21:82-8.

2. Mubarak AE. Nutritional composition and antinutritional factors of mung bean seeds (Phaseolus aureus) as affected by some home traditional processes. Food Chem. 2005;89:489-95.

3. Li SZ. Part of cereals. In: Wang YJ, editor. Ben-Cao-Gang-Mu. Beijing: People's Hygiene Publishing; 1999. p. 1344-9.

4. Kakumanu A, Ambavaram MM, Klumas C, Krishnan A, Batlang U, Myers E, et al. Effects of drought on gene expression in maize reproductive and leaf meristem tissue revealed by RNA-Seq. Plant Physiol. 2012;160:846-67.

5. Nelson SK, Oliver MJ. A soil-plate based pipeline for assessing cereal root growth in response to polyethylene glycol (PEG)-induced water deficit stress. Front Plant Sci. 2017;8:1272. 
6. Marok MA, Tarrago L, Ksas B, Henri P, Abrous-Belbachir O, Havaux M, et al. A drought-sensitive barley variety displays oxidative stress and strongly increased contents in low-molecular weight antioxidant compounds during water deficit compared to a tolerant variety. J Plant Physiol. 2013;170:633-45.

7. Noctor G, Mhamdi A, Foyer $\mathrm{CH}$. The roles of reactive oxygen metabolism in drought: Not so cut and dried. Plant Physiol. 2014;164:1636-48.

8. Zhu K, Cui W, Dai C, Wu M, Zhang J, Zhang Y, et al. Methane-rich water alleviates $\mathrm{NaCl}$ toxicity during alfalfa seed germination. Environ Exp Bot. 2016:129:37-47.

9. Liu D, Xu S, Hu H, Pan J, Li P, Shen W. Endogenous hydrogen sulfide homeostasis is responsible for the alleviation of senescence of postharvest daylily flower via increasing antioxidant capacity and maintained energy status. J Agric Food Chem. 2017;65:718-26.

10. Conrad R. Soil microorganisms as controllers of atmospheric trace gases $\left(\mathrm{H}_{2}, \mathrm{CO}, \mathrm{CH}_{4}, \mathrm{OCS}, \mathrm{N}_{2} \mathrm{O}\right.$, and NO). Microbiol Rev. 1996;60:609-40.

11. Xie Y, Mao Y, Lai D, Zhang W, Zheng T, Shen W. Roles of NIA/NR/ NOA1-dependent nitric oxide production and HY1 expression in modulation of Arabidopsis salt tolerance. J Exp Bot. 2013;64:3045-60.

12. Su J, Zhang $Y$, Nie $Y$, Cheng D, Wang $R$, Hu H, et al. Hydrogen-induced osmotic tolerance is associated with nitric oxide-mediated proline accumulation and reestablishment of redox balance in alfalfa seedlings. Environ Exp Bot. 2018;147:249-60

13. Jin Q, Zhu K, Cui W, Xie Y, Han B, Shen W. Hydrogen gas acts as a novel bioactive molecule in enhancing plant tolerance to paraquat-induced oxidative stress via the modulation of heme oxygenase-1 signalling system. Plant Cell Environ. 2013;36:956-69.

14. Rodríguez-Ruiz M, Mateos RM, Codesido V, Corpas FJ, Palma JM. Characterization of the galactono-1,4-lactone dehydrogenase from pepper fruits and its modulation in the ascorbate biosynthesis. Role of nitric oxide. Redox Biol. 2017;12:171-81.

15. Wang TT, Shi ZQ, Hu LB, Xu XF, Han FX, Zhou LG, et al. Thymol ameliorates cadmium-induced phytotoxicity in the root of rice (Oryza sativa) seedling by decreasing endogenous nitric oxide generation. J Agric Food Chem. 2017:65:7396-405.

16. Besson-Bard A, Pugin A, Wendehenne D. New insights into nitric oxide signaling in plants. Annu Rev Plant Biol. 2008;59:21-39.

17. García-Mata C, Lamattina L. Nitric oxide induces stomatal closure and enhances the adaptive plant responses against drought stress. Plant Physiol. 2001;126:1196-204.

18. Tian $X$, Lei $Y$. Nitric oxide treatment alleviates drought stress in wheat seedlings. Biol Plant. 2006;50:775-8.

19. Zhang LP, Mehta SK, Liu ZP, Yang ZM. Copper-induced proline synthesis is associated with nitric oxide generation in Chlamydomonas reinhardtii. Plant Cell Physiol. 2008;49:411-9.

20. Tossi V, Lamattina L, Cassia R. An increase in the concentration of abscisic acid is critical for nitric oxide-mediated plant adaptive responses to UV-B irradiation. New Phytol. 2009;181:871-9.

21. Tripathi DK, Mishra RK, Singh S, Singh S, Vishwakarma K, Sharma S, et al. Nitric oxide ameliorates zinc oxide nanoparticles phytotoxicity in wheat seedlings: implication of the ascorbate-glutathine cycle. Front Plant Sci. 2017:8:1.

22. Tripathi DK, Singh S, Singh S, Srivastava PK, Singh VP, Singh S, et al. Nitric oxide alleviates silver nanoparticles (AgNps)-induced phytotoxicity in Pisum sativum seedlings. Plant Physiol Biochem. 2017;110:167-77.

23. Delledonne M, Xia Y, Dixon RA, Lamb C. Nitric oxide functions as a signal in plant disease resistance. Nature. 1998;394:585-8.

24. Samalova M, Johnson J, Illes M, Kelly S, Fricker M, Gurr S. Nitric oxide generated by the rice blast fungus Magnaporthe oryzae drives plant infection. New Phytol. 2013;197:207-22.

25. Ninnemann $\mathrm{H}$, Maier J. Indication for the occurrence of nitric oxide synthase in fungi and plants and the involvement in photoconidiation of Neurospora crassa. Photoch Photobiol. 1996;64:393-8.

26. Bethke PC, Badger MR, Jones RL. Apoplastic synthesis of nitric oxide by plant tissues. Plant Cell. 2004;16:332-41.

27. Yu SX, Feng QN, Xie HT, Li S, Zhang Y. Reactive oxygen species mediate tapetal programmed cell death in tobacco and tomato. BMC Plant Biol. 2017; 17:76.

28. Lindermayr C, Saalbach G, Durner J. Proteomic identification of Snitrosylated proteins in Arabidopsis. Plant Physiol. 2005;137:921-30.
29. Yun BW, Feechan A, Yin M, Saidi NBB, Le Bihan T, Yu M, et al. S-nitrosylation of NADPH oxidase regulates cell death in plant immunity. Nature. 2011;478:264-8.

30. Ghyczy M, Torday C, Kaszaki J, Szabó A, Czóbel M, Boros M. Oral phosphatidylcholine pretreatment decreases ischemia-reperfusion-induced methane generation and the inflammatory response in the small intestine. Shock. 2008;30:596-602.

31. Boros M, Ghyczy M, Érces D, Varga G, Tőkés T, Kupai K, et al. The antiinflammatory effects of methane. Crit Care Med. 2012;40:1269-78.

32. Chen O, Ye Z, Cao Z, Manaenko A, Ning K, Zhai X, et al. Methane attenuates myocardial ischemia injury in rats through anti-oxidative, anti-apoptotic and anti-inflammatory actions. Free Radic Biol Med. 2016;90:1-11.

33. Qaderi MM, Reid DM. Methane emissions from six crop species exposed to three components of global climate change: temperature, ultraviolet-B radiation and water stress. Physiol Plant. 2009;137:139-47.

34. Bruhn D, Mikkelsen TN, Øbro J, Willats WGT, Ambus P. Effects of temperature, ultraviolet radiation and pectin methyl esterase on aerobic methane release from plant material. Plant Biology. 2009;11:43-8.

35. Bloom AA, Lee-Taylor J, Madronich S, Messenger DJ, Palmer PI, Reay DS, et al. Global methane emission estimates from ultraviolet irradiation of terrestrial plant foliage. New Phytol. 2010;187:417-25.

36. Wang ZP, Gulledge J, Zheng JQ, Liu W, Li LH, Han XG. Physical injury stimulates aerobic methane emissions from terrestrial plants. Biogeosciences. 2009;6:615-21.

37. Keppler F, Hamilton JTG, Braß M, Röckmann T. Methane emissions from terrestrial plants under aerobic conditions. Nature. 2006:439:187-91.

38. Cui W, Qi F, Zhang Y, Cao H, Zhang J, Wang R, Shen W. Methane-rich water induces cucumber adventitious rooting through heme oxygenase1/carbon monoxide and $\mathrm{Ca}^{2+}$ pathways. Plant Cell Rep. 2015;34:435-45.

39. Qi F, Xiang Z, Kou N, Cui W, Xu D, Wang R, et al. Nitric oxide is involved in methane-induced adventitious root formation in cucumber. Physiol Plant. 2017;159:366-77.

40. Samma MK, Zhou H, Cui W, Zhu K, Zhang J, Shen W. Methane alleviates copper-induced seed germination inhibition and oxidative stress in Medicago sativa. BioMetals. 2017;30:97-111.

41. Han B, Duan X, Wang Y, Zhu K, Zhang J, Wang R, et al. Methane protects against polyethylene glycol-induced osmotic stress in maize by improving sugar and ascorbic acid metabolism. Sci Rep. 2017;7:46185.

42. Puntarulo S, Sánchez RA, Boveris A. Hydrogen peroxide metabolism in soybean embryonic axes at the onset of germination. Plant Physiol. 1988;86:626-30.

43. Liptay A, Schopfer P. Effect of water stress, seed coat restraint, and abscisic acid upon different germination capabilities of two tomato lines at low temperature. Plant Physiol. 1983;73:935-8.

44. Xuan W, Xu S, Li M, Han B, Zhang B, Zhang J, et al. Nitric oxide is involved in hemin-induced cucumber adventitious rooting process. J Plant Physiol. 2012;169:1032-9.

45. Xie Y, Mao Y, Zhang W, Lai D, Wang Q, Shen W. Reactive oxygen speciesdependent nitric oxide production contributes to hydrogen-promoted stomatal closure in Arabidopsis. Plant Physiol. 2014;165:759-73.

46. Han Y, Zhang J, Chen X, Gao Z, Xuan W, Xu S, et al. Carbon monoxide alleviates cadmium-induced oxidative damage by modulating glutathione metabolism in the roots of Medicago sativa. New Phytol. 2008;177:155-66.

47. Aslam M, Huffaker RC. Dependency of nitrate reduction on soluble carbohydrates in primary leaves of barley under aerobic conditions. Plant Physiol. 1984;75:623-8

48. Paleg LG. Physiological effects of gibberellic acid: I. On carbohydrate metabolism and amylase activity of barley endosperm. Plant Physiol. 1960;35:293-9.

49. Zhao MG, Chen L, Zhang LL, Zhang WH. Nitric reductase-dependent nitric oxide production is involved in cold acclimation and freezing tolerance in Arabidopsis. Plant Physiol. 2009;151:755-67.

50. Zhao MG, Tian QY, Zhang WH. Nitric oxide synthase-dependent nitric oxide production is associated with salt tolerance in Arabidopsis. Plant Physiol. 2007;144:206-17.

51. Sun A, Wang W, Ye $X$, Wang $Y$, Yang $X$, Ye Z, et al. Protective effective of methane-rich saline on rats with lipopolysaccharide-induced acute lung injury. Oxid Med Cell Longe. 2017:7430193. 